\title{
Variación espacial y temporal en la dispersión de polen en un huerto semillero y en rodales naturales cercanos de Pinus patula
}

\author{
Temporal and spatial variation of pollen dispersal in a seed orchard \\ and nearby natural stands of Pinus patula
}

\author{
Liliana Muñoz-Gutiérrez a , J Jesús Vargas-Hernández a*, Javier López-Upton a, \\ Carlos Ramírez-Herrera a, Marcos Jímenez-Casas a, Arnulfo Aldrete a , Ramón Díaz-Ruíz ${ }^{\text {b }}$

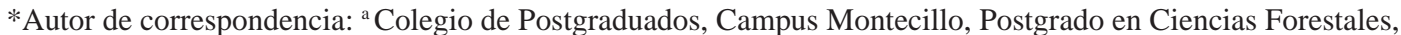 \\ Carretera México-Texcoco, km 36,5, Montecillo, Texcoco CP 56230, Estado de México, México, \\ tel.: 01 (595) 9520200 Ext. 1469, vargashj@colpos.mx

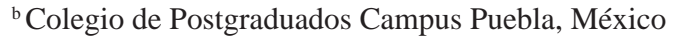

\begin{abstract}
SUMMARY
Pollen flow from natural populations towards seed orchards has a negative effect on the genetic quality of germplasm produced. The objective of the study was to evaluate the potential gene flow into a Pinus patula clonal seed orchard from nearby natural stands. In 2014 and 2015, pollen traps were established at the center and periphery of the seed orchard and in nearby natural stands along an altitudinal transect in Aquixtla, Puebla, Mexico. With the pollen capture curves, total pollen dispersal (pollen grains $\mathrm{cm}^{-2}$ ), dates and extent of the dispersal period were estimated. The relationship of the dispersal period with temperature, rainfall and degree-days was also analyzed. Pollen captured in the seed orchard was 1.5 times higher and dispersal started one week earlier than in natural stands in both years. In 2015, the amount of pollen dispersal doubled and the dispersion period was delayed. The pollen captured at the center of the orchard was $74 \%$ higher than in the periphery, and the dispersal period started one to three days later. No altitudinal trend in the dispersal period was observed in natural stands. Phenology differences between years in pollen dispersal were related to cumulative degree-days and to variation in maximum temperature and rainfall. Results reveal risks of genetic contamination in the seed orchard, though these are lower in the center than in the periphery.
\end{abstract}

Key words: genetic contamination, reproductive phenology, gene flow, elevation gradient, degree-day.

\section{RESUMEN}

El flujo de polen de poblaciones naturales a huertos semilleros tiene un efecto negativo en la calidad genética del germoplasma producido. El objetivo del estudio fue evaluar el potencial de flujo genético de rodales naturales vecinos a un huerto semillero clonal de Pinus patula. En 2014 y 2015 se establecieron trampas para capturar polen en el centro y periferia del huerto y en rodales naturales en un transecto altitudinal en Aquixtla, Puebla, México. Con las curvas de polen capturado se estimó el total dispersado por unidad de superficie (granos de polen $\mathrm{cm}^{-2}$ ), las fechas y la duración del periodo de dispersión. También se analizó la relación del periodo de dispersión con la temperatura, precipitación y grados-día. El polen capturado en el huerto fue 1,5 veces mayor y el periodo de dispersión inició una semana antes que en los rodales naturales en ambos años. En 2015, el número de granos de polen $\mathrm{cm}^{-2}$ aumentó al doble y el periodo de dispersión se retrasó. En la parte central del huerto hubo 74 \% más polen que en la periferia y su periodo de dispersión se retrasó uno a tres días. En los rodales naturales no se observó un patrón altitudinal en la dispersión de polen. Las diferencias entre años en la fenología de dispersión se relacionaron con los grados-día acumulados y con la variación en temperatura máxima y precipitación. Los resultados señalan riesgos de contaminación genética en el huerto, pero estos son menores en el centro que en la periferia.

Palabras clave: contaminación genética, fenología reproductiva, flujo genético, gradiente altitudinal, grados-día.

\section{INTRODUCCIÓN}

Los programas de mejoramiento genético forestal generalmente requieren del establecimiento de huertos semilleros que constituyen el vínculo para transferir a la producción forestal la ganancia genética obtenida en la po- blación de mejora (Lowe y Wheeler 1993). En los huertos semilleros se obtiene el germoplasma necesario para producir las plantas de calidad genética que se requieren en los programas de reforestación y plantaciones comerciales más productivas y adaptadas a su ambiente (Di-Giovanni y Kevan 1991). Los huertos semilleros se pueden estable- 
cer por semilla (huertos sexuales) o mediante propágulos vegetativos (huertos asexuales o clonales) de individuos seleccionados con base en la superioridad en algunas características importantes (Chmura et al. 2012).

El objetivo final del huerto es producir semilla de mayor calidad, en cantidad suficiente y con una eficiencia genética elevada, la cual depende del tamaño efectivo de población en el huerto y de la capacidad de exclusión de gametos de individuos no seleccionados (Wang et al. 1991). Factores como la fecundidad de los progenitores, sincronía en la fenología reproductiva de los clones y ausencia de polen externo influyen en la eficiencia genética del huerto (Lowe y Wheeler 1993). Estas condiciones son difíciles de cumplir en especies de coníferas (Squillace y Long 1981, Adams y Burczyk 2000).

El polen externo de árboles no seleccionados que llega a los huertos semilleros tiene efectos negativos en la progenie porque reduce el potencial de crecimiento y genera problemas de adaptación que reducen la ganancia genética (Lowe y Wheeler 1993). Debido a la capacidad de producción y dispersión natural del polen en especies anemófilas, es común que sus huertos semilleros se expongan a altos niveles de polen externo, a pesar de que se encuentren relativamente alejados de poblaciones de la misma especie (Friedman y Adams 1985). En huertos semilleros de Pinus sylvestris L. se ha encontrado que el nivel de contaminación genética puede variar de 2 a 74 \% (El-Kassaby et al. 1989). En otras especies, los valores de contaminación generalmente son superiores a $40 \%$ como en Pinus taeda L. (Adams y Burczyk 2000) y Picea abies (L.) H. Karst (Pakkanen et al. 2000), aunque pueden variar desde menos de $10 \%$, como en Pseudotsuga menziesii (Mirb.) Franco (Ritland y El-Kassaby 1985), a más de $85 \%$, como en Pinus brutia Ten. (Kaya et al. 2006).

El potencial de contaminación de polen externo en un huerto semillero depende del grado de aislamiento (físico, fenológico o por distancia) con respecto a otras fuentes no deseadas de polen, y de la capacidad de producción de polen en el huerto, que compita con la fuente externa (DiGiovanni y Kevan 1991). El primer factor está asociado con la capacidad y el momento de dispersión del polen; la distancia de dispersión varía en función de condiciones ambientales, como dirección y velocidad del viento, precipitación y humedad relativa (Lindgren et al. 1995), y de características de la especie como morfología y tamaño de los granos de polen (Sousa y Hattemer 2003).

En poblaciones continuas hay un flujo extenso de polen que puede ser alrededor del 4 o $5 \%$ en distancias de hasta $100 \mathrm{~km}$ (Kremer et al. 2012). Este flujo es suficiente para evitar la diferenciación y la estructuración de las poblaciones en regiones extensas, ya que se requieren pocos migrantes por generación para contrarrestar los efectos de la selección natural y la deriva genética en la estructura espacial de las poblaciones (Hedrick 1985). Sin embargo, en un estudio de dispersión de polen de 36 árboles en un rodal aislado de Pinus sylvestris se ha demostrado que al- rededor de $50 \%$ del polen producido por un árbol cae en una distancia no mayor a $11 \mathrm{~m}$, y $7 \%$ cae fuera de una distancia de 200 m (Robledo-Arnuncio y Gil 2005). Por lo tanto, las diferencias en polen capturado en diferentes sitios dentro de una población continua reflejan en gran medida las diferencias en producción de polen en los vecindarios muestreados.

El momento de la liberación del polen es afectado por la temperatura, la humedad y los grados-calor o grados-día acumulados durante el proceso de diferenciación y desarrollo posterior de las estructuras reproductivas (Huusko y Hicks 2009). Por lo anterior, se espera que el periodo de producción y dispersión del polen varíe de un año a otro en vecindarios o subpoblaciones a lo largo de un transecto altitudinal (Adams 1992). Por otro lado, la capacidad de producción de polen en un huerto semillero, y el grado de competencia con el polen externo, depende de la edad y tamaño de copa en los individuos dentro del huerto (Caron y Powell 1989, Di-Giovanni y Kevan 1991). Las coníferas inician la producción de polen después de varios años de crecimiento, pero una vez iniciada, su capacidad aumenta gradualmente con la edad (Caron y Leblanc 1992). Debido a esto, el potencial de contaminación por polen externo generalmente es elevado en huertos jóvenes en donde apenas inicia la producción de polen, pero este se reduce conforme aumenta la edad de los árboles en el huerto (Caron y Powell 1989).

En el año 2003 se estableció un huerto semillero clonal de Pinus patula Schiede ex Schltdl. et Cham., en la Sierra Norte de Puebla, como parte de un programa regional de mejoramiento genético de la especie, para satisfacer las necesidades de semilla con calidad genética superior para el establecimiento de plantaciones forestales (Castaños y Castro-Zavala 2014). El huerto se ubica dentro del área de distribución natural de la especie, a una elevación de $2.860 \mathrm{~m}$, en la parte media de un transecto altitudinal, en donde existen rodales naturales de P. patula, por lo que existe un riesgo potencial de contaminación genética. En este trabajo se evalúa la cantidad de polen presente dentro del huerto y en rodales naturales vecinos. Los objetivos son a) estimar la variación espacial y temporal en la dispersión de polen dentro del huerto de $P$. patula y en rodales naturales de la misma especie cercanos al huerto a lo largo de un transecto altitudinal, y b) determinar si la variación en el periodo de dispersión del polen de un año a otro a lo largo del transecto altitudinal está relacionada con las condiciones meteorológicas y grados-día acumulados. Las hipótesis son: 1) la cantidad de polen dispersado en el huerto es mayor que en los rodales naturales, pero el periodo de dispersión coincide en ambos; 2) la cantidad de polen dispersado y el periodo de dispersión dentro del huerto no difieren espacialmente; 3) el periodo de dispersión de polen en rodales naturales vecinos se retrasa al aumentar la elevación del sitio; 4) el periodo de dispersión de polen difiere de un año a otro en función de los gradosdía acumulados. 


\section{MÉTODOS}

Área de estudio. El estudio se realizó en un huerto semillero asexual de Pinus patula de 10 años de edad, establecido mediante injertos en la Reserva Forestal Multifuncional "El Manantial", en el municipio de Aquixtla, Puebla, México (19०43' 13" N, $97^{\circ} 59^{\prime} 20^{\prime \prime}$ O, 2.860 m s.n.m.). El huerto cubre una superficie de 1,2 ha, en un diseño completamente al azar con un espaciamiento de $3 \times 3 \mathrm{~m}$ con diferente número de rametos (1-22) por clon. Al inicio del estudio el huerto contaba con 660 rametos pertenecientes a 82 clones distintos. También se muestrearon cuatro rodales naturales coetáneos de la especie a lo largo de un transecto altitudinal (2.807-3.011 m) a una distancia de 150 a 1.000 $\mathrm{m}$ del centro del huerto (cuadro 1); al menos el $50 \%$ de los árboles adultos (en edad reproductiva) se encontraba produciendo polen. Para fines del estudio el huerto semillero y los rodales naturales se consideraron como dos poblaciones distintas.

Localización de trampas y recolecta de polen. Para cuantificar la variación espacial y temporal en la presencia de polen se colocaron trampas de captura en diferentes sitios dentro y fuera del huerto. En el huerto se establecieron cinco sitios de muestreo, uno en el centro y cuatro en la periferia, a una distancia de 9 a $12 \mathrm{~m}$ del borde, cada uno con cuatro trampas; fuera del huerto también se establecieron cuatro trampas de muestreo en cada uno de los rodales naturales $\left(\mathrm{R}_{1}-\mathrm{R}_{4}\right)$ a lo largo del transecto altitudinal mencionado.

Las trampas consistieron en portaobjetos recubiertos con cinta adhesiva doble cara colocados en una pequeña plataforma con una veleta, montada en tubos de PVC a una altura de $6 \mathrm{~m}$ sobre el nivel del suelo, que representa la altura de la parte media de la copa de los árboles en el huerto. En cada sitio las trampas se colocaron con una separación horizontal de un metro entre ellas. Las muestras (portaobjetos) se cambiaron cada 72 horas en todos los sitios de muestreo durante el periodo de dispersión de polen en cada año de evaluación. El conteo de granos de polen de $P$. patula retenidos en las trampas se hizo directamente en cada portaobjeto utilizando un microscopio óptico (Zeizz M 40) con aumento 40X. El portaobjetos se dividió en ochos secciones de un $\mathrm{cm}^{2}$, y cuatro de estas secciones se muestrearon en forma de zigzag. Aunque no es posible distinguir el origen o procedencia del polen capturado en las trampas, de acuerdo con los datos de Robledo-Arnuncio y Gil (2005), la mayor proporción del polen presente en un punto dentro de una población extensa procede de un vecindario cercano (radio $\leq 75 \mathrm{~m}$ ), por lo que bajo este supuesto y considerando una proporción homogénea de flujo de polen dentro de la población, las diferencias en el polen capturado por las trampas en los sitios reflejan las diferencias en producción de polen entre los vecindarios muestreados.

Información meteorológica. Durante el periodo de estudio (2014-2015) la información meteorológica se obtuvo de tres micro-estaciones automatizadas, establecidas en 2009 a diferentes elevaciones dentro de la Reserva Forestal, cercanas a los sitios de muestreo y captura de polen. La primera de ellas se encuentra en la zona central del huerto, la segunda en la parte alta del transecto a una elevación de 3.000 m, (estación Sierra Mojada), cercana a los sitios $\mathrm{R}_{3}$ y $\mathrm{R}_{4}$ y la tercera en la parte baja del transecto (estación Chichicaxtla) a una altitud de $2.660 \mathrm{~m}$, cercana al sitio $\mathrm{R}_{1}$. Las variables registradas en las micro-estaciones fueron temperatura máxima, mínima y media por día y precipitación diaria en los meses previos y durante el periodo de dispersión del polen de los ciclos 2014 y 2015.

Para calcular los grados-día (GD) en los ciclos 20142015 se utilizaron las temperaturas máximas y mínimas diarias registradas desde el primer día de enero en cada año hasta el fin del periodo de dispersión. Dado que en la estación Chichicaxtla no se registraron las temperaturas máximas y mínimas diarias durante el periodo del estudio, estas se estimaron a través de regresión lineal utilizando los datos registrados en las otras dos micro-estaciones en los años 2011 y 2012. Los grados-día (GD) acumulados se calcularon con la siguiente ecuación:

Cuadro 1. Localización y características generales de los rodales naturales de Pinus patula a lo largo de un transecto altitudinal en los que se muestreó la dispersión de polen.

Location and general conditions of Pinus patula natural stands along the altitudinal transect where pollen dispersal was sampled.

\begin{tabular}{|c|c|c|c|c|c|c|c|c|}
\hline Rodal & $\begin{array}{l}\text { Latitud } \\
\text { (N) }\end{array}$ & $\begin{array}{l}\text { Longitud } \\
\text { (O) }\end{array}$ & $\begin{array}{c}\text { Altitud } \\
\text { (m s.n.m.) }\end{array}$ & $\begin{array}{c}\text { Densidad } \\
(\text { árboles ha-1) }\end{array}$ & $\begin{array}{c}\text { Edad } \\
\text { (años) }\end{array}$ & $\begin{array}{l}\text { Diámetro } \\
\text { medio }(\mathrm{cm})\end{array}$ & $\begin{array}{l}\text { Altura media } \\
\text { (m) }\end{array}$ & $\begin{array}{c}\text { Distancia } \\
\text { huerto (m) }\end{array}$ \\
\hline $\mathrm{R}_{1}$ & $19^{\circ} 44^{\prime} 05,4^{\prime \prime}$ & $97^{\circ} 59^{\prime} 06,9 ”$ & 2.807 & 400 & 45 & 40 & 21 & 850 \\
\hline $\mathrm{R}_{2}$ & $19^{\circ} 43^{\prime} 51,2^{\prime \prime}$ & $97^{\circ} 59^{\prime} 12,6^{\prime \prime}$ & 2.870 & 750 & 26 & 24 & 19 & 150 \\
\hline $\mathrm{R}_{3}$ & $19^{\circ} 43^{\prime} 36,6^{\prime \prime}$ & $97^{\circ} 59^{\prime} 29,1^{\prime \prime}$ & 3.011 & 900 & 20 & 18 & 16 & 750 \\
\hline $\mathrm{R}_{4}$ & $19^{\circ} 43^{\prime} 24,7^{\prime \prime}$ & $97^{\circ} 59^{\prime} 36,8^{\prime \prime}$ & 3.009 & 1.500 & 16 & 15 & 13 & 1.000 \\
\hline
\end{tabular}




$$
\mathrm{GD}=\left[\frac{\mathrm{T}^{\circ} \mathrm{máx}+\mathrm{T}^{\circ} \mathrm{mín}}{2}\right]-\mathrm{T}^{\circ} \text { umbral }
$$

donde: $T_{\text {máx }}^{\circ}=$ temperatura máxima diaria $\left({ }^{\circ} \mathrm{C}\right), T^{\circ}{ }_{\text {min }}=$ temperatura mínima diaria $\left({ }^{\circ} \mathrm{C}\right)$ y $T^{\circ}{ }_{\text {umbral }}=$ temperatura umbral inferior $\left({ }^{\circ} \mathrm{C}\right)$. Se utilizó una temperatura umbral de $6{ }^{\circ} \mathrm{C}$, con base en el trabajo realizado por Salaya-Domínguez et al. (2012) para la misma especie y lugar de estudio; cuando los valores de GD fueron negativos con la fórmula empleada, estos se consideraron como cero.

Variables evaluadas y análisis estadístico. A partir de los datos de polen capturado en cada trampa se estimó la cantidad de polen de P. patula por centímetro cuadrado así como la fecha de inicio y terminación (número de días a partir del 1 de enero) y la duración del periodo de dispersión del polen; también se estimó la fecha en que se alcanzó el $50 \%$ del total de polen dispersado en el periodo. Las trampas se consideraron como repeticiones o muestras independientes en cada sitio. Para cada trampa se generó una curva acumulada de dispersión de polen por ciclo reproductivo (2014 y 2015), a partir de la cual se determinaron las fechas en que se alcanzó 5 y $95 \%$ del total de polen dispersado en el periodo, que se consideraron como fechas de inicio y terminación del periodo de dispersión, respectivamente. El día en que se alcanzó $50 \%$ del polen dispersado en el periodo se estimó de la misma curva.

El análisis de varianza para determinar la variación espacial y temporal en la dispersión del polen se realizó con el procedimiento Mixed del paquete estadístico SAS (SAS Institute 2002). En una primera etapa se analizaron las diferencias en la cantidad de polen (granos $\mathrm{cm}^{-2}$ ) y fechas del periodo de dispersión entre el huerto semillero y los rodales naturales en los dos ciclos reproductivos considerando los valores promedio de cada sitio de muestreo como repeticiones, obtenidos a partir de los valores promedio de las cuatro trampas establecidas en cada sitio o rodal. Se utilizó el siguiente modelo estadístico, en el que todos los factores se consideraron de efectos fijos, excepto el error:

$$
Y_{i j k}=\mu+A_{i}+P_{j}+A_{i} P_{j}+\varepsilon_{i j k}
$$

donde: $\mathrm{Y}_{\mathrm{ijk}}=$ valor promedio del $\mathrm{k}$-ésimo sitio en la j-ésima población [huerto semillero o rodal natural] en el i-ésimo año; $\mu=$ media general; $\mathrm{A}_{\mathrm{i}}=$ efecto del i-ésimo año de evaluación; $\mathrm{P}_{\mathrm{j}}=$ efecto de la j-ésima población; $\mathrm{A}_{\mathrm{i}} \mathrm{P}_{\mathrm{j}}=\mathrm{l}$ efecto de la interacción del i-ésimo año con la j-ésima población; y $\varepsilon_{\mathrm{ijk}}=$ error.

En una etapa posterior se analizó la variación espacial dentro del huerto y a lo largo del transecto altitudinal en los rodales naturales por separado. En todos los análisis los factores año y zona (o rodal) se consideraron como efectos fijos. Los datos de conteo de granos de polen fueron transformados con la raíz cuadrada del valor original previo al análisis, para homogeneizar las varianzas y ajustar los residuales a una distribución normal; después del análisis, los valores promedio obtenidos fueron re-transformados a su escala original.

\section{RESULTADOS}

Cantidad y periodo de dispersión de polen en el huerto semillero y en los rodales naturales. Todas las variables evaluadas presentaron diferencias significativas $(P \leq 0,05)$ entre años y entre poblaciones (huerto semillero vs. rodales naturales), excepto la fecha de terminación del periodo de dispersión que no mostró diferencias entre el huerto y los rodales. La fecha en que se alcanzó 50 \% de dispersión del polen fue la única variable con efecto significativo de la interacción año x población (cuadro 2).

La cantidad promedio de polen (granos $\mathrm{cm}^{-2}$ ) aumentó al doble en 2015 con respecto a 2014; de la misma manera, el polen presente en el huerto semillero, en promedio de los dos años de evaluación, fue 2,5 veces mayor que en los rodales naturales vecinos (cuadro 3, figura 1). En 2015 el periodo de dispersión de polen se retrasó en el huerto semillero y en los rodales naturales; la dispersión inició nueve días

Cuadro 2. Significancia estadística $(P)$ para cantidad de polen, fechas y duración del periodo de dispersión de polen en las poblaciones (huerto semillero y rodales naturales) de Pinus patula.

Statistical significance $(P)$ for total pollen grains, dates and length of the pollen dispersal period in the seed orchard and natural stands of

\begin{tabular}{|c|c|c|c|c|c|}
\hline \multirow[t]{2}{*}{ Fuente de variación } & \multirow{2}{*}{$\begin{array}{c}\text { Polen } \\
\left(\text { granos } \mathrm{cm}^{-2} \text { ) }\right.\end{array}$} & \multicolumn{3}{|c|}{$\begin{array}{l}\text { Fechas del periodo de dispersión } \\
\text { (días a partir del } 1 \text { de enero) }\end{array}$} & \multirow{2}{*}{$\begin{array}{l}\text { Duración } \\
\text { (días) }\end{array}$} \\
\hline & & inicio & $50 \%$ & fin & \\
\hline Año & 0,006 & $<0,001$ & $<0,001$ & $<0,001$ & 0,002 \\
\hline Población $^{\dagger}$ & $<0,001$ & $<0,001$ & 0,008 & 0,128 & $<0,001$ \\
\hline Año x Población & 0,703 & 0,075 & 0,030 & 0,905 & 0,065 \\
\hline
\end{tabular}
Pinus patula.

${ }^{\dagger}$ El huerto semillero y los rodales naturales se consideraron como poblaciones diferentes. 
Cuadro 3. Valores promedio de cantidad de polen, fechas y duración de la dispersión en el huerto semillero y en rodales naturales de Pinus patula. Valores promedio dentro de cada factor seguidos por la misma letra no son estadísticamente diferentes $($ Tukey, $P<0,05)$.

Mean values for number of pollen grains, dates and length of dispersal period in the seed orchard and in natural stands of Pinus patula. Mean values within each factor followed by the same letter are not statistically different (Tukey, $P<0.05$ ).

\begin{tabular}{|c|c|c|c|c|c|}
\hline \multirow[t]{2}{*}{ Factor y niveles } & \multirow{2}{*}{$\begin{array}{c}\text { Polen } \\
\text { (granos } \mathrm{cm}^{-2} \text { ) }\end{array}$} & \multicolumn{3}{|c|}{$\begin{array}{l}\text { Fechas del periodo de dispersión } \\
\text { (días a partir del } 1 \text { de enero) }\end{array}$} & \multirow{2}{*}{$\begin{array}{l}\text { Duración } \\
\text { (días) }\end{array}$} \\
\hline & & Inicio & $50 \%$ & fin & \\
\hline \multicolumn{6}{|l|}{ Año } \\
\hline 2014 & $877 \mathrm{~b}$ & $64,9 \mathrm{~b}$ & $72,8 \mathrm{~b}$ & $77,2 \mathrm{~b}$ & $12,3 \mathrm{~b}$ \\
\hline 2015 & $1.716 \mathrm{a}$ & 73,6 a & 86,7 a & 89,9 a & 16,3 a \\
\hline \multicolumn{6}{|l|}{ Población } \\
\hline Huerto semillero & $1.883 \mathrm{a}$ & $65,3 \mathrm{~b}$ & $79,0 \mathrm{~b}$ & 83,2 a & 17,9 a \\
\hline Rodales naturales & $764 \mathrm{~b}$ & 73,2 a & 80,5 a & 83,9 a & $10,6 \mathrm{~b}$ \\
\hline
\end{tabular}
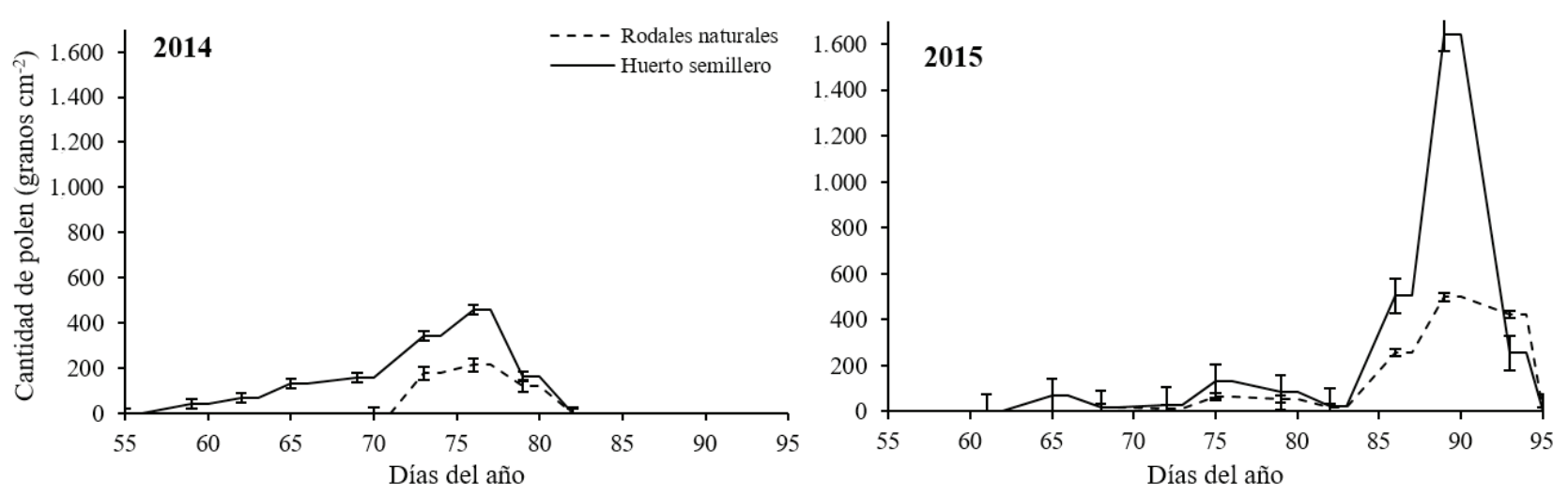

Figura 1. Dispersión de polen en el huerto semillero y en rodales naturales de Pinus patula para los ciclos reproductivos 2014 y 2015. Las barras verticales representan el error estándar de los valores promedio en cada fecha de muestreo.

Pollen dispersal in the seed orchard and in natural stands (along an altitudinal gradient) of Pinus patula for 2014 and 2015 reproductive cycles. Vertical bars represent the standard error of mean values at each sampling date.

después y terminó 13 días después, por lo que el periodo de dispersión se alargó cuatro días en el segundo año (16,3 vs 12,3 días). En 2014, ocho días después del inicio se alcanzó $50 \%$ del total dispersado, mientras que en 2015 ocurrió 13 días después. En el huerto la dispersión de polen inició en promedio ocho días antes que en los rodales naturales, pero el $50 \%$ de la dispersión se alcanzó solo dos días antes y el final ocurrió casi al mismo tiempo que en los rodales naturales, por lo que la dispersión de polen en el huerto tuvo una duración mayor (18 vs. 11 días en promedio). La diferencia entre huerto y rodales en la fecha en que se alcanzó $50 \%$ de dispersión fue mayor en 2014 que en 2015 (figura 1).

Variación espacial y temporal en la dispersión del polen en el huerto semillero. Todas las variables evaluadas, ex- cepto la duración del periodo de dispersión, mostraron diferencias significativas $(P \leq 0,05)$ entre años y entre zonas del huerto semillero. Ninguna de las variables mostró efectos significativos de la interacción año x zona (cuadro 4). La cantidad de polen dispersado aumentó al doble en 2015 con respecto a 2014 (3.008 vs 1.536 granos $\mathrm{cm}^{-2}$ ). De manera similar, la cantidad de polen capturado en la parte central del huerto fue $74 \%$ mayor que en la periferia en los dos años (cuadro 5, figura 2).

El inicio y fin de la dispersión de polen se retrasó 12 días en promedio en el ciclo 2015 con respecto al ciclo 2014, mientras que la fecha en que se alcanzó $50 \%$ de dispersión total se retrasó en 15 días, del día 72 en 2014, al día 87 en 2015 (cuadro 5). Sin embargo, la duración del periodo de dispersión de polen fue similar en ambos 
Cuadro 4. Significancia estadística $(P)$ para cantidad de polen, fechas y duración del periodo de dispersión de polen en las zonas del huerto semillero de Pinus patula.

Statistical significance $(P)$ for total pollen grains, dates and length of pollen dispersal period in zones within the Pinus patula seed orchard.

\begin{tabular}{|c|c|c|c|c|c|}
\hline \multirow{2}{*}{ Fuente de variación } & \multirow{2}{*}{$\begin{array}{c}\text { Polen } \\
(\text { granos cm-2) }\end{array}$} & \multicolumn{3}{|c|}{$\begin{array}{l}\text { Fechas del periodo de dispersión } \\
\text { (días a partir del } 1 \text { de enero) }\end{array}$} & \multirow{2}{*}{$\begin{array}{l}\text { Duración } \\
\text { (días) }\end{array}$} \\
\hline & & inicio & $50 \%$ & fin & \\
\hline Año & $<0,001$ & $<0,001$ & $<0,001$ & $<0,001$ & 0,242 \\
\hline Zona & $<0,001$ & 0,027 & 0,030 & 0,043 & 0,083 \\
\hline Año x Zona & 0,200 & 0,251 & 0,282 & 0,532 & 0,157 \\
\hline
\end{tabular}

Cuadro 5. Valores promedio de cantidad de polen, fechas y duración de la dispersión por año y zona en el huerto semillero de Pinus patula. Valores promedio dentro de cada factor seguidos por la misma letra no son estadísticamente diferentes (Tukey, $P<0,05)$.

Mean values for number of pollen grains, dates and length of dispersal period by year and zone in the Pinus patula seed orchard. Mean values within each factor followed by the same letter are not statistically different (Tukey, $P<0.05$ ).

\begin{tabular}{|c|c|c|c|c|c|}
\hline \multirow[t]{2}{*}{ Factor y niveles } & \multirow{2}{*}{$\begin{array}{c}\text { Polen } \\
\left(\text { granos } \mathrm{cm}^{-2}\right)\end{array}$} & \multicolumn{3}{|c|}{$\begin{array}{l}\text { Fechas del periodo de dispersión } \\
\text { (días a partir del } 1 \text { de enero) }\end{array}$} & \multirow{2}{*}{$\begin{array}{l}\text { Duración } \\
\text { (días) }\end{array}$} \\
\hline & & inicio & $50 \%$ & fin & \\
\hline \multicolumn{6}{|l|}{ Año } \\
\hline 2014 & $1.536 \mathrm{~b}$ & $60,2 \mathrm{~b}$ & $71,9 \mathrm{~b}$ & $77,1 \mathrm{~b}$ & 16,9 a \\
\hline 2015 & $3.008 \mathrm{a}$ & 71,8 a & 86,7 a & 89,8 a & 18,0 a \\
\hline \multicolumn{6}{|l|}{ Zona del huerto } \\
\hline Centro & $2.863 \mathrm{a}$ & $67,1 \mathrm{a}$ & 79,9 a & 83,8 a & 16,6 a \\
\hline Periferia & $1.643 \mathrm{~b}$ & $64,8 \mathrm{~b}$ & $78,8 \mathrm{~b}$ & $83,1 \mathrm{~b}$ & 18,3 a \\
\hline
\end{tabular}

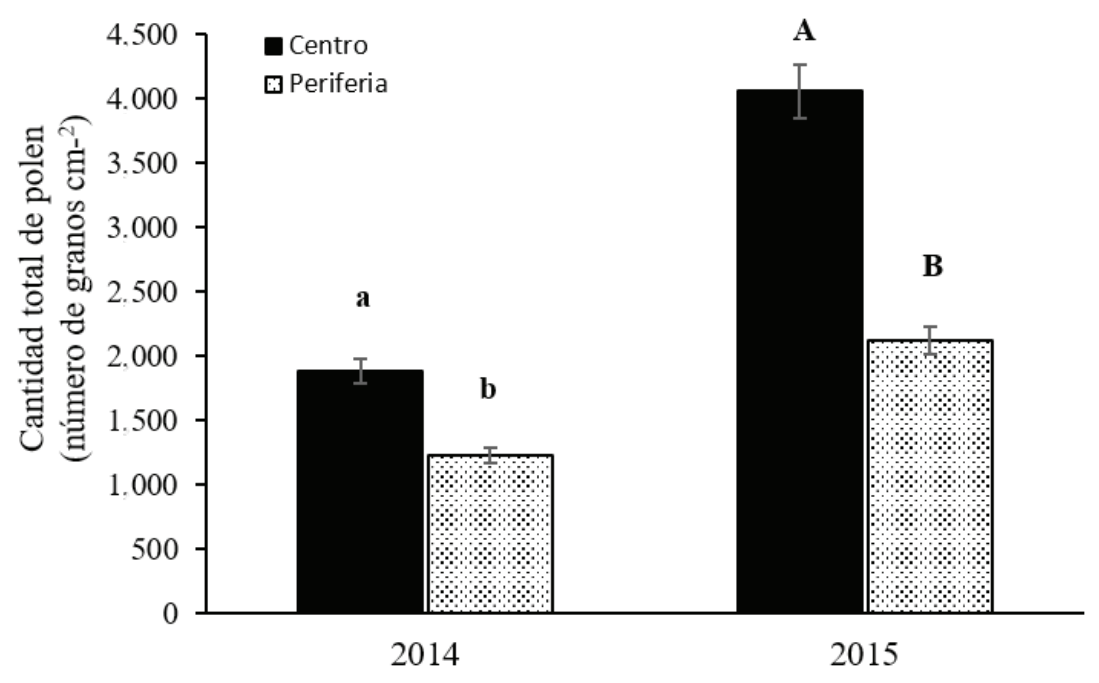

Figura 2. Cantidad de polen (granos de polen $\mathrm{cm}^{-2}$ ) capturado en la zona centro (C) y periferia (P) del huerto semillero de Pinus patula en 2014 y 2015 . Columnas dentro de un año de evaluación con distinta letra indica diferencias estadísticas $($ Tukey, $P<0,05)$ entre zonas del huerto semillero.

Total pollen (pollen grains $\mathrm{cm}^{-2}$ ) captured at the center and periphery zones of the Pinus patula seed orchard in 2014 and 2015. Bars within each sampling year with different letter indicate statistical differences (Tukey, $P<0.05$ ) among seed orchard zones. 
años (17 vs 18 días). Las diferencias entre zonas en las fechas de dispersión de polen fueron menores, con uno a tres días de adelanto en el inicio, $50 \%$ y fin del periodo de dispersión del polen en la periferia con respecto al centro del huerto (cuadro 5).

Variación espacial y temporal en la dispersión de polen en los rodales naturales. Todas las variables mostraron diferencias significativas $(P \leq 0,05)$ entre los ciclos reproductivos, pero no entre rodales a lo largo del transecto altitudinal, excepto la fecha de terminación del periodo de dispersión (cuadro 6). Por otro lado, la interacción año $\mathrm{x}$ rodal fue significativa $(P \leq 0,05)$ en las fechas de inicio, fin y duración del periodo de dispersión, pero no en la cantidad de polen ni en la fecha en que se alcanzó $50 \%$ de dispersión (cuadro 6).

$\mathrm{Al}$ igual que en el huerto semillero, la cantidad de polen capturado en los rodales naturales a lo largo del transecto en 2015 fue el doble que en 2014 (cuadro 7), aunque con valores menores que en el huerto (1.032 vs 497 granos $\mathrm{cm}^{-2}$ ). De la misma manera, en 2015 el periodo de dispersión inició siete días después, mientras que las fechas en que se alcanzó 50 \% y finalizó la dispersión se retrasaron en 12-13 días con respecto a 2014; debido a ello, el periodo de dispersión en 2015 aumentó en seis días (cuadro 7). Además, en 2014 la fecha en que se alcanzó $50 \%$ de la dispersión ocurrió cuatro días después del inicio, mientras que en 2015 fue 10 días después, generando una curva de dispersión distinta.

En los rodales sólo se encontraron diferencias significativas en la fecha de terminación del periodo de dispersión; los rodales $\mathrm{R}_{2}$ y $\mathrm{R}_{4}$ terminaron uno y dos días antes que los rodales $\mathrm{R}_{1} \mathrm{y} \mathrm{R}_{3}$. Sin embargo, la interacción año x rodal fue significativa para las fechas de inicio y terminación y para la duración del periodo, por lo que la fenología de la dispersión del polen a lo largo del transecto altitudinal varió de un año a otro. En 2014 se observaron diferencias de hasta cuatro días en la fecha de inicio de la dispersión del

Cuadro 6. Significancia estadística $(P)$ para cantidad de polen, fechas y duración del periodo de dispersión de polen en rodales naturales de Pinus patula a lo largo de un transecto altitudinal.

Statistical significance $(P)$ for total pollen grains, dates and length of pollen dispersal period in natural stands of Pinus patula along an altitudinal transect.

\begin{tabular}{lcrrrr}
\hline \multirow{2}{*}{ Fuente de variación } & \multirow{2}{*}{$\begin{array}{c}\text { Polen } \\
\left.\text { (granos cm }{ }^{-2}\right)\end{array}$} & \multicolumn{3}{c}{$\begin{array}{c}\text { Fechas del periodo de dispersión } \\
\text { (días a partir del 1 de enero) }\end{array}$} & \multirow{2}{*}{$\begin{array}{c}\text { Duración } \\
\text { (días) }\end{array}$} \\
\cline { 3 - 5 } & $<0,001$ & inicio & $50 \%$ & fin & \\
\hline Año & 0,413 & 0,001 & $<0,001$ & $<0,001$ & $<0,001$ \\
Rodal & 0,127 & 0,380 & 0,015 & 0,351 \\
Año x Rodal & 0,281 & 0,004 & 0,057 & 0,003 & 0,019 \\
\hline
\end{tabular}

Cuadro 7. Valores promedio de cantidad de polen, fechas y duración del periodo de dispersión por año y rodal de Pinus patula a lo largo de un transecto altitudinal. Valores promedio dentro de cada factor seguidos por la misma letra no son estadísticamente diferentes (Tukey, $P<0,05)$.

Mean values for number of pollen grains, dates and length of dispersal period by year and stand of Pinus patula along an altitudinal transect. Mean values within each factor followed by the same letter are not statistically different (Tukey, $P<0.05$ ).

\begin{tabular}{|c|c|c|c|c|c|}
\hline \multirow{2}{*}{$\begin{array}{l}\text { Año y rodal } \\
\text { (altitud) }\end{array}$} & \multirow{2}{*}{$\begin{array}{c}\text { Polen } \\
\text { (granos } \mathrm{cm}^{-2} \text { ) }\end{array}$} & \multicolumn{3}{|c|}{$\begin{array}{l}\text { Fechas del periodo de dispersión } \\
\quad \text { (días a partir del } 1 \text { de enero) }\end{array}$} & \multirow{2}{*}{$\begin{array}{l}\text { Duración } \\
\text { (días) }\end{array}$} \\
\hline & & inicio & $50 \%$ & fin & \\
\hline \multicolumn{6}{|l|}{ Año } \\
\hline 2014 & $497 \mathrm{~b}$ & $69,9 \mathrm{~b}$ & $74,3 \mathrm{~b}$ & $77,6 \mathrm{~b}$ & 7,6 b \\
\hline 2015 & $1.032 \mathrm{a}$ & 76,6 a & 86,9 a & 90,3 a & 13,7 a \\
\hline \multicolumn{6}{|l|}{ Rodal (altitud) } \\
\hline $\mathrm{R}_{1}(2.807 \mathrm{~m})$ & 771 a & 74,0 a & 80,8 a & $84,1 \mathrm{ab}$ & $10,1 \mathrm{a}$ \\
\hline $\mathrm{R}_{2}(2.870 \mathrm{~m})$ & 864 a & $71,4 \mathrm{a}$ & 79,9 a & 83,3 b & 11,9 a \\
\hline $\mathrm{R}_{3}(3.011 \mathrm{~m})$ & 756 a & 73,9 a & 81,0 a & 84,9 a & 11,0 a \\
\hline $\mathrm{R}_{4}(3.009 \mathrm{~m})$ & 585 a & $73,8 \mathrm{a}$ & 80,6 a & $83,4 \mathrm{~b}$ & 9,6 a \\
\hline
\end{tabular}


polen entre los rodales, pero sin un patrón claro a lo largo del transecto altitudinal (figura 3); en cambio, las fechas de $50 \%$ y fin de la dispersión mostraron un patrón altitudinal, con un retraso de uno a dos días conforme aumentó la altitud del sitio (figura 3). En 2015 se observaron diferencias mayores (4-5 días) entre rodales en la fecha de inicio que en las fechas de $50 \%$ y fin del periodo de dispersión, pero en ningún caso se observó el mismo patrón altitudinal que en 2014 (figura 3).
Relación del periodo de dispersión de polen con las variables meteorológicas y grados-día. La temperatura (gradosdía) y la precipitación influyeron sobre el patrón estacional de dispersión del polen en el huerto en los dos años de evaluación. El periodo de dispersión en ambos años inició cuando se acumularon entre 255-260 GD contados a partir del 1 de enero, mientras que el $50 \%$ de dispersión se alcanzó con 330 GD y el final ocurrió al acumularse entre 340 y 370 GD (figura 4A).

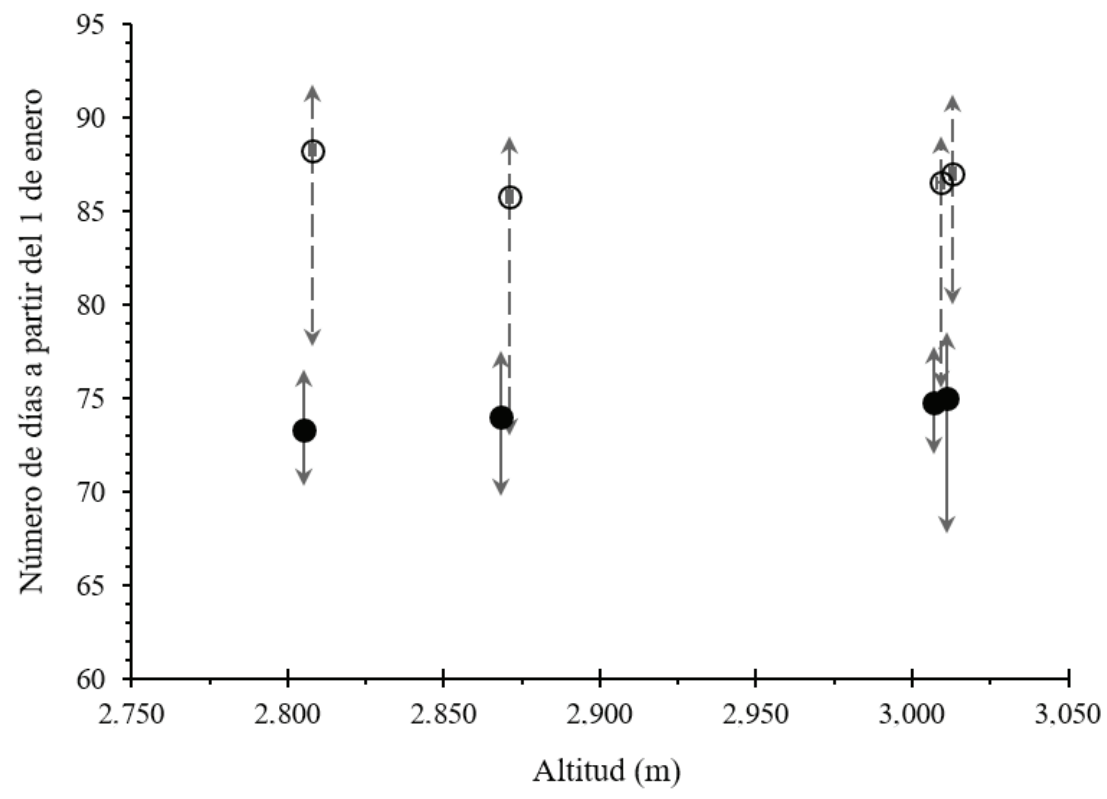

Figura 3. Periodo de dispersión de polen en rodales naturales de Pinus patula a lo largo del transecto altitudinal durante los ciclos reproductivos 2014 (línea continua) y 2015 (línea discontinua). Las puntas de las flechas indican el inicio y el fin del periodo y el círculo indica la fecha en que se alcanzó 50 \% de dispersión. Las líneas en 2015 están ligeramente desfasadas en altitud para evitar su traslape con las líneas de 2014.

Pollen dispersal period in natural stands of Pinus patula along an altitudinal transect during 2014 (continuous line) and 2015 (dashed line) reproductive cycles. The arrowheads indicate the onset and end of the period and the circle shows the date when $50 \%$ of pollen dispersal was reached. Lines for 2015 are slightly moved up in elevation to avoid overlapping them with lines in 2014.
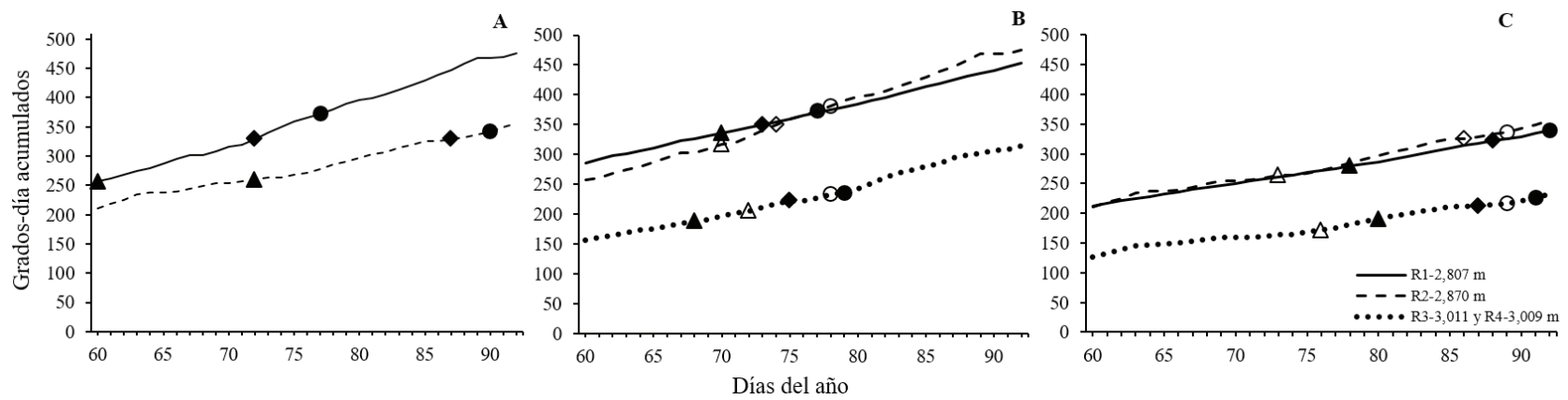

Figura 4. Relación del periodo de dispersión del polen con los grados-dia acumulados en los ciclos 2014 (línea continua) y 2015 (línea discontinua) en el huerto semillero de Pinus patula (A) y en los rodales naturales a lo largo del transecto altitudinal para los ciclos 2014 (B) y 2015 (C). Los simbolos a lo largo de las líneas indican las fechas de inicio $(\boldsymbol{\Delta}, \Delta), 50 \%(\diamond, \diamond)$ y fin $(\bullet, \circ)$ del periodo de dispersión.

Relationship of the pollen dispersal period with the cumulative degree-days in 2014 (continuous line) and 2015 (dashed line) reproductive cycles in the Pinus patula seed orchard (A) and in natural stands along the altitudinal transect for cycles 2014 (B) and 2015 (C). Symbols along the lines indicate the onset $(\boldsymbol{\Delta}, \Delta), 50 \%(\diamond, \diamond)$, and end $(\bullet, \circ)$ dates of pollen dispersal period. 
En los rodales naturales a lo largo del transecto, los periodos de dispersión ocurrieron a diferentes GD acumulados. En el ciclo 2014, la dispersión de polen en los rodales $\mathrm{R}_{1}$ y $\mathrm{R}_{2}$ inició cuando se acumularon entre 315 y $335 \mathrm{GD}$, mientras que en los rodales de mayor altitud $\left(\mathrm{R}_{3}\right.$ y $\left.\mathrm{R}_{4}\right)$ ocurrió cuando se acumularon entre 185 y 205 GD (figura 4B). Para el ciclo 2015, en los rodales $\mathrm{R}_{1}$ y $\mathrm{R}_{2}$ la dispersión inició al acumularse entre 260 y $280 \mathrm{GD}$, mientras que en $\mathrm{R}_{3}$ y $\mathrm{R}_{4}$ ocurrió con 170 y $190 \mathrm{GD}$ (figura 4C). El final del periodo de dispersión mostró un patrón altitudinal similar con respecto a los años de evaluación (figura 4B y 4C).
Las diferencias entre años en el periodo de dispersión de polen en el huerto se relacionaron también con diferencias en temperatura y precipitación en los días antes y durante la dispersión (figura 5A y 5B). La mayor amplitud en el periodo de dispersión del polen en 2015 se relacionó con una temperatura máxima más baja y una precipitación abundante, en particular durante los días previos e inicio del periodo de dispersión.
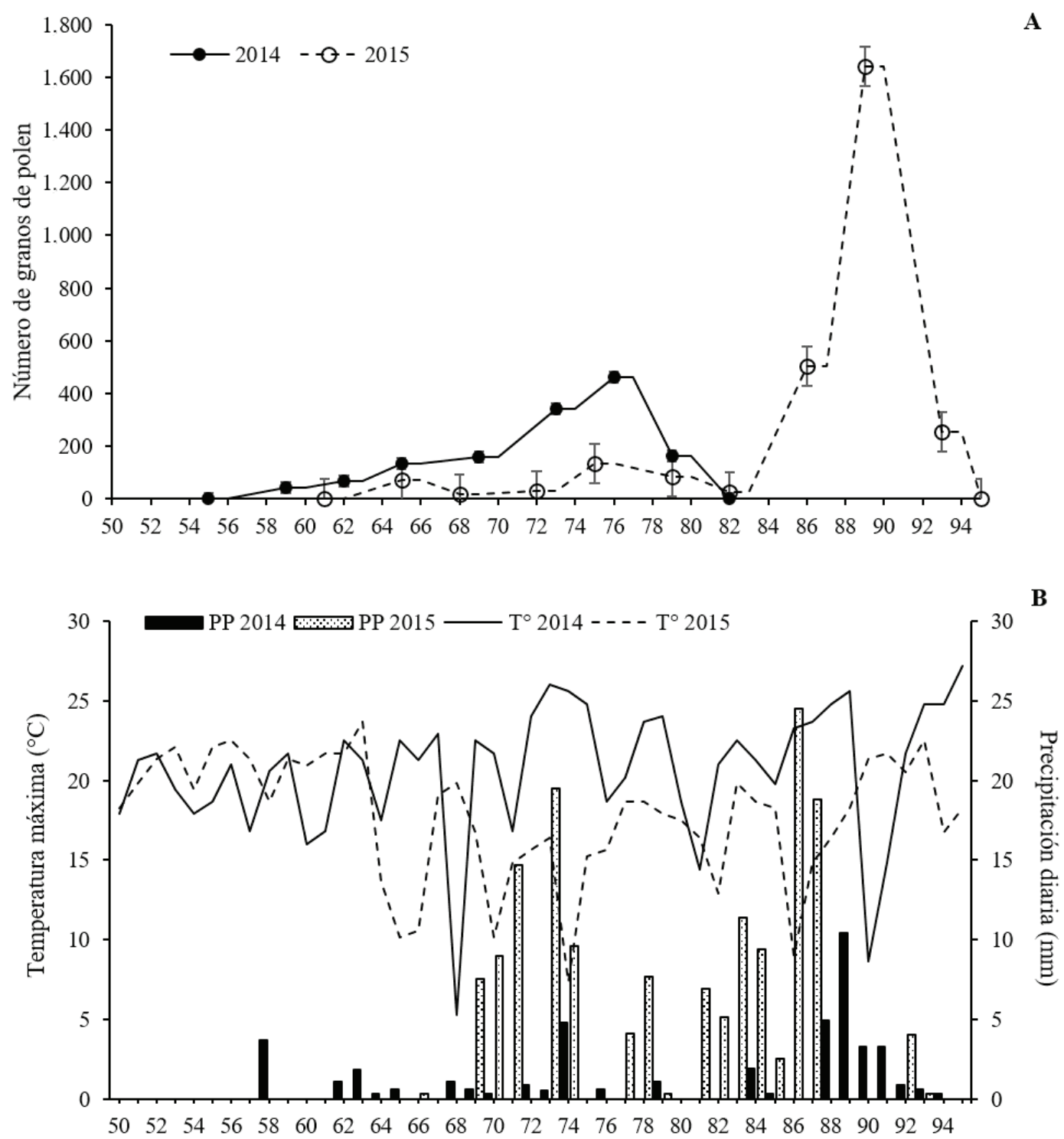

Días del año

Figura 5. A) Curva de dispersión de polen en el huerto semillero de Pinus patula, con base en los granos de polen capturados en las trampas durante los ciclos 2014 (línea continua) y 2015 (línea discontinua); y B) temperatura máxima $\left({ }^{\circ} \mathrm{C}\right.$ ) y precipitación (mm) diarias registradas durante el periodo de dispersión en 2014 y 2015.

A) Pollen dispersal curve in the Pinus patula seed orchard, based on pollen grains captured in traps during the 2014 (continuous line) and 2015 (dashed line) reproductive cycles; B) maximum daily temperature $\left({ }^{\circ} \mathrm{C}\right)$ and rainfall $(\mathrm{mm})$ recorded during the pollen dispersal period in 2014 and 2015. 


\section{DISCUSIÓN}

Dispersión del polen en el huerto semillero y en los rodales naturales. La capacidad de producción de polen en el huerto semillero de Pinus patula difiere con respecto a los rodales naturales vecinos, lo cual puede influir sobre el potencial de contaminación genética por polen externo, como se ha demostrado en otras especies (Caron y Leblanc 1992). La mayor cantidad de granos de polen por unidad de superficie encontrada en el huerto puede estar asociada a diferencias en vigor de los árboles y en densidad de población con respecto a los rodales naturales, ya que se trata de individuos más jóvenes en edad reproductiva (injertos de 10-11 años de edad en el huerto vs 15-45 años de edad en los rodales naturales, y con una mayor densidad poblacional (1.130 árboles ha-1 en el huerto vs. 400-1.500 árboles ha $\mathrm{a}^{-1}$ en los rodales). Estos resultados difieren de los descritos por Kaya et al. (2006) en un huerto semillero de Pinus brutia de 11 años de edad, en donde la cantidad de polen producido es menor que en rodales naturales disetáneos, con árboles de hasta 100 años de edad. Wang et al. (1991) también encontraron una producción baja de polen en huertos de $P$. sylvestris de 25-27 años de edad.

La producción mayor de polen en los individuos del huerto de $P$. patula con respecto a los rodales naturales fue consistente en los dos años de estudio, a pesar de la variación natural en la producción de polen en diferentes ciclos reproductivos de las especies forestales. Caron y Powell (1989) demostraron que la producción de polen en huertos jóvenes de Picea mariana (Mill.) Britton, Sterns et Poggenb., incrementa año con año conforme aumenta la edad de los árboles. Esto hace suponer que la producción de polen en el huerto puede contrarrestar el impacto del flujo de fuentes externas de polen y esta capacidad aumentará con la edad de los árboles en el huerto cuando estos desarrollen copas más grandes, con un número mayor de estróbilos masculinos y mayor interferencia física al ingreso de polen de fuentes cercanas al huerto, como se ha mencionado para otras especies (Caron y Leblanc 1992).

Las diferencias fenológicas en el periodo de dispersión del polen entre el huerto semillero y los rodales naturales también reducen los riesgos de contaminación por polen externo (Di-Giovanni y Kevan 1991, Caron y Leblanc 1992). En los dos años de evaluación se encontró, de manera consistente, que el periodo de dispersión de polen en el huerto inició al menos una semana antes que en los rodales naturales, pero con un traslape amplio, ya que la dispersión se prolongó en el huerto y terminó casi en la misma fecha que en los rodales naturales (cuadro 3, figura 1). Las diferencias en el inicio y duración del periodo de dispersión se debieron, al menos en parte, a la menor edad de los árboles en el huerto y a la variación genética en fenología reproductiva de los clones representados en este (Hernández-Zaragoza et al. 2016). La dispersión de polen en huertos semilleros jóvenes puede ocurrir antes que en rodales naturales, porque en árboles jóvenes el pe- riodo de crecimiento del brote generalmente inicia antes y en los huertos el espaciamiento es mayor (Torimaru et al. 2009). Además, la parte baja de la copa de los árboles jóvenes, donde se desarrollan los estróbilos masculinos, está cercana al suelo donde la temperatura es mayor, lo que puede acelerar el desarrollo de los estróbilos masculinos (Lindgren et al. 1995), como se demostró en huertos de Pinus sylvestris (Harju y Nikkanen 1996). En Picea abies se observó que los primeros dos días de dispersión la cantidad de polen en el huerto fue similar a la de los rodales naturales vecinos y posteriormente aumentó dentro del huerto, debido a diferencias en la fenología por el origen geográfico de los clones (Nikkanen et al. 2002).

Sin embargo, no hubo diferencias importantes en el periodo de dispersión de polen entre los rodales naturales (cuadros 5 y 6), a pesar de que uno de ellos tenía árboles de 15 a 20 años de edad. Esto indica que la variación genética en la fenología reproductiva de los clones es un factor importante en el inicio y duración del periodo de dispersión del polen en el huerto. Un estudio realizado antes en el huerto muestra que hay una amplia variación fenológica entre los clones en el desarrollo y maduración de los estróbilos masculinos (Hernández-Zaragoza et al. 2016). Los clones que se encuentran en el huerto provienen de árboles superiores seleccionados en los rodales naturales de la especie en la región, por lo que se espera que la variación genética en el huerto sea mayor que en cada uno de los rodales naturales cercanos, como se ha demostrado en Pseudotsuga menziesii (Erickson y Adams 1989) y en Picea abies (Nikkanen et al. 2002). El mosaico de heterogeneidad ambiental en áreas de montaña hace que pequeñas diferencias en el origen de los clones generen variación en la fenología reproductiva entre éstos (Khanduri 2012).

Variación espacial y temporal en la dispersión del polen dentro del huerto. La cantidad mayor de polen y el retraso en el periodo de dispersión en el centro del huerto con respecto a la periferia (cuadro 5) pueden estar asociados a un efecto de "borde", con un menor número de árboles en el vecindario y cambios en el microambiente suficientes para modificar ligeramente la fenología de la dispersión del polen. Nikkanen et al. (2002) también encontraron una amplia variación en la densidad de polen en diferentes zonas de un huerto semillero de Picea abies, la que atribuyeron a factores asociados con la densidad del arbolado, el tamaño del huerto, la dirección del viento y diferencias fenológicas en la dispersión de polen en los vecindarios dentro del huerto.

Friedman y Adams (1985) enfatizan la importancia de la producción y distribución espacial del polen en huertos de Pinus taeda, especialmente en los árboles que se encuentran cerca del borde o perímetro, ya que estos están expuestos a una mayor cantidad de polen foráneo que los del centro. En el huerto de P. patula, la menor cantidad de polen en la periferia indica que hay menores posibilidades de competir con el polen externo; aunque el periodo 
de dispersión se adelantó ligeramente, la diferencia no es suficiente para eliminar el periodo de traslape con la producción de polen en los rodales vecinos. En P. sylvestris, Harju y Nikkanen (1996) encontraron que los estróbilos femeninos estuvieron receptivos antes de la dispersión de polen en el huerto, lo que implica un riesgo alto de contaminación, aunque la dispersión del polen en los rodales naturales de la misma especie usualmente inicia más tarde que en el huerto.

Relación entre la fenología de la dispersión de polen y variables meteorológicas. En Pseudotsuga menziesii (Erickson y Adams 1989) y Pinus radiata D. Don (Codesido et al. 2005) se ha encontrado que la duración del periodo de dispersión de polen depende de las condiciones meteorológicas en cada ciclo reproductivo. En este estudio, las diferencias encontradas entre años en la fenología de la dispersión del polen, tanto en el huerto como en los rodales naturales a lo largo del transecto altitudinal, se asociaron con las condiciones meteorológicas, en particular con la acumulación de grados-día (figura 4), de manera consistente a lo que se reporta para Picea mariana (Caron y Leblanc 1992) y $P$. radiata (Codesido et al. 2005). En los estudios antes mencionados, el inicio de la floración se correlacionó positivamente con los grados-día acumulados en la etapa previa de crecimiento. Esto indica que el tiempo requerido para iniciar el periodo de floración está bajo un fuerte control genético y fisiológico; la floración está sincronizada con la suma de grados-día (Boes et al. 1991) ya que la temperatura influye sobre la velocidad de desarrollo de las estructuras reproductivas. Codesido et al. (2005) indican que para iniciar los procesos de floración, receptividad femenina y dispersión de polen se requiere una cierta cantidad de temperatura acumulada. La cantidad menor de grados-día requeridos en los dos rodales situados a mayor altitud en el transecto altitudinal sugiere el valor adaptativo de esta característica en las poblaciones naturales de la especie y explica, en parte, el periodo mayor de dispersión de polen en el huerto y la mayor variación en la fenología reproductiva de los clones. En Pseudotsuga menziesii encontraron que a una altitud de $305 \mathrm{~m}$ la dispersión de polen inicia cuando se alcanzan 800-900 horas-día por arriba de $10{ }^{\circ} \mathrm{C}$, pero a altitudes entre 762 y $1.067 \mathrm{~m}$ requiere sólo 400 horas-día (Ebell y Schmidt 1964).

La temperatura y la precipitación también influyen en el inicio y duración del periodo de dispersión del polen, así como en la dinámica de dispersión de un año a otro; estos dos factores están asociados con la acumulación de grados-día, pero también influyen sobre la humedad relativa de la atmósfera (Boes et al. 1991). Por ello, la menor temperatura y mayor precipitación durante los días previos y al inicio del periodo de dispersión en 2015 (figura 5) posiblemente redujeron la dispersión del polen y ampliaron el periodo. En especies con dispersión anemófila, como los pinos, una alta humedad relativa en el ambiente reduce la apertura de las escamas en los estróbilos y limita la cantidad de polen liberado y su distancia de dispersión como se ha observado en $P$. sylvestris (Chmura et al. 2012) y $P$. roxburghii Sarg. (Khanduri 2012). Nikkanen et al. (2002) mencionan la presencia de picos en la curva de dispersión de polen en Picea abies asociados a la reducción de la humedad relativa en el ambiente. En cambio, la presencia de lluvias frecuentes durante el periodo de dispersión ocasiona la pérdida de polen y suspende temporalmente la dispersión (Caron y Leblanc 1992, Codesido et al. 2005).

Implicaciones sobre el riesgo de contaminación genética en la progenie del huerto. A pesar de que el huerto semillero de $P$. patula inicia la dispersión del polen antes que los rodales vecinos, el amplio traslape en el periodo de dispersión en ambas poblaciones indica que en las condiciones actuales existe riesgo de que el polen externo llegue al huerto y fecunde los óvulos, reduciendo la calidad genética del germoplasma. El riesgo de contaminación genética es mayor en la zona periférica del huerto debido a la menor cantidad de polen que se encontró en esta zona y a su exposición mayor al flujo de polen externo. El aumento en el número de copas de los árboles conforme se avanza al centro del huerto constituye una barrera física que limita la cantidad de polen foráneo que puede llegar (Nikkanen et al. 2002). Este factor, aunado a la producción mayor de polen en el centro del huerto, sugiere que las posibilidades de contaminación en esa zona son menores, similar a lo encontrado por Nikkanen et al. (2002) en Picea abies. Sin embargo, el riesgo de contaminación genética disminuye conforme aumenta la capacidad de producción de polen en el huerto, como se reportó en huertos semilleros de Picea mariana (Caron y Leblanc 1992).

Debido a la ubicación del huerto semillero con respecto a poblaciones naturales y plantaciones de Pinus patula en los predios cercanos, en la práctica es imposible aislar físicamente al huerto del flujo de polen externo. Sin embargo, la relación encontrada entre la dispersión del polen y las variables meteorológicas, en particular la suma de grados-día y la humedad, así como el inicio anticipado del periodo de dispersión en el huerto en relación con los rodales vecinos, ofrece la posibilidad de adelantar la fenología reproductiva en los árboles del huerto aumentando la temperatura, como se ha sugerido en otras especies para reducir los daños por heladas y acelerar el desarrollo de las estructuras reproductivas (Pakkanen et al. 2000). Por otro lado, los riesgos de contaminación genética detectados sugieren la necesidad de evaluar otros aspectos de la fenología reproductiva en el huerto semillero, como la variación clonal en la dispersión del polen y receptividad de los óvulos en rametos, y el grado de sincronía en estos procesos (Codesido et al. 2005). También es necesario realizar un análisis de paternidad en una muestra de la progenie del huerto para determinar el nivel real de contaminación genética, con base en las técnicas desarrolladas en otras especies (Kaya et al. 2006, Torimaru et al. 2009). 


\section{CONCLUSIONES}

En el huerto de Pinus patula hay mayor cantidad de polen que en los rodales naturales de manera consistente en los dos años evaluados; el polen dispersado aumenta en el segundo año. La dispersión de polen en el huerto inicia antes que en los rodales naturales, pero presenta un periodo de dispersión más amplio, lo que genera un fuerte traslape en el periodo de dispersión del polen dentro y fuera del huerto. La dispersión de polen es mayor en el centro que en la periferia y ocurre con uno a tres días de retraso, lo cual sugiere que el riesgo de contaminación genética por polen externo difiere en estas zonas dentro del huerto.

Hay diferencias entre rodales en la fecha de terminación de dispersión del polen, pero el periodo de dispersión no muestra un patrón claro asociado con la elevación de los rodales naturales dentro del intervalo altitudinal muestreado. Sin embargo, la relación fenológica de la dispersión del polen con los grados-día acumulados en los dos años de evaluación en el huerto y en los rodales naturales sugiere una relación adaptativa en la que la dispersión del polen requiere una cantidad menor de grados-día al aumentar la elevación. Debido a los riesgos de contaminación genética que existen en el huerto de Pinus patula y a la dificultad para aislarlo físicamente de las poblaciones naturales vecinas, se recomienda implementar prácticas de manejo en el huerto para adelantar su periodo de floración, aumentar la producción de polen y su efectividad en la fecundación de los óvulos.

\section{AGRADECIMIENTOS}

Al Ing. León Jorge Castaños Martínez y al Ing. Salvador Castro Zavala, propietarios de la Reserva Forestal Multifuncional “El Manantial”, por las facilidades y apoyo logístico proporcionado durante la realización del estudio. Al CONACyT por la beca proporcionada a Liliana $\mathrm{Mu}-$ ñoz-Gutiérrez para la realización de sus estudios de doctorado en ciencias y al Colegio de Postgraduados por el apoyo financiero otorgado para la realización de este trabajo a través de la Línea Prioritaria de Investigación "Manejo Sustentable de Recursos Naturales".

\section{REFERENCIAS}

Adams WT, J Burczyk. 2000. Magnitude and implications of gene flow in gene conservation reserves. In Young A, D Boshier, T Boyle eds. Forest Conservation Genetics: Principles and Practice. Collingwood, Victoria, Australia. CSIRO Publishing. p. 215-224.

Adams WT. 1992. Gene dispersal within forest tree populations. New Forests 6: 217-240.

Boes TK, JR Brandle, WR Lovett. 1991. Characterization of flowering phenology and seed yield in a Pinus sylvestris clonal seed orchard in Nebraska. Canadian Journal of Forest Research 21: 1721-1729.

Caron EG, R Leblanc. 1992. Pollen contamination in a small black spruce seedling seed orchard for 3 consecutive years. Forest Ecology and Management 53: 245-261.

Caron GE, GR Powell. 1989. Patterns of seed-cone and pollencone production in young Picea mariana trees. Canadian Journal of Forest Research 19: 359-364.

Chmura DJ, R Rozkowski, W Chalupka. 2012. Growth and phenology variation in progeny of Scots pine seed orchards and commercial seed stands. European Journal of Forest Research 131: 1229-1243.

Codesido V, E Merlo, J Fernández-López. 2005. Variation in reproductive phenology in a Pinus radiata D. Don seed orchard in northern Spain. Silvae Genetica 54: 246-256.

Di-Giovanni P, PG Kevan. 1991. Factors affecting pollen dynamics and its importance to pollen competition: a review. Canadian Journal of Forest Research 21: 1151-1170.

Ebell LF, RL Schmidt. 1964. Meteorological factors affecting conifer pollen dispersal on Vancouver Island. Government of Canada, Department of Forestry, Forest Entomology and Pathology Branch, Ottawa. Department of Forestry Publication No. 1036. 34 p.

El-Kassaby YA, D Rudin, R Yazdani. 1989. Levels of outcrossing and contamination in two Pinus sylvestris L. seed orchards in northern Sweden. Scandinavian Journal of Forest Research 4: 41-49.

Ericksson VJ, WT Adams. 1989. Mating success in a costal Douglas-fir seed orchard as affected by distance and floral phenology. Canadian Journal of Forest Research 19: 1248-1255.

Friedman ST, WT Adams. 1985. Estimation of gene flow into two seed orchards of loblolly pine (Pinus taeda L.). Theoretical and Applied Genetics 69: 609-615.

Harju A, T Nikkanen. 1996. Reproductive success of orchard and nonorchard pollens during different stages of pollen shedding in Scots pine seed orchard. Canadian Journal of Forest Research 26: 1096-1102.

Hedrick PW. 1985. Genetics of Populations. Boston, Massachusetts, USA. Jones and Bartlett Publishers. 629 p.

Hernández-Zaragoza O, J López-Upton, JJ Vargas-Hernández, M Jiménez-Casas. 2016. Variación clonal de la fenología reproductiva en un huerto semillero de Pinus patula. Bosque 37(2): 255-264.

Kaya N, K Isik, WT Adams. 2006. Mating system and pollen contamination in a Pinus brutia seed orchard. New Forests 31: 409-416.

Khanduri PV. 2012. Temporal and spatial variation of pollen yield in natural populations of Pinus roxburghii. Forestry Studies in China 14: 20-29.

Kremer A, O Ronce, JJ Robledo-Arnuncio, F Guillaume, G Bohrer, R Nathan, JR Bridle, R Gomuñkiewicz, EK Klein, K Ritland, A Kuparinen, S Gerber, S Schueler. 2012. Long distance gene flow and adaptation of forest trees to rapid climate change. Ecology Letters 15: 378-392.

Lindgren D, L Paule, S Xihuan, R Yazdani, U Segerström, JE Wallin, ML Lejdebro. 1995. Can viable pollen carry Scots pine genes over long distances? Grana 34: 64-69.

Lowe WJ, NC Wheeler. 1993. Pollen contamination in seed orchards. In Bramlett DL, GR Askew, TD Blush, FE Bridgwater, JB Jett eds. Advances in pollen management. Agriculture Handbook 698. USDA Forest Service. p. 49-53.

Nikkanen T, A Pakkanen, J Heinonen. 2002. Temporal and spatial variation in airbone pollen and quality of the seed crop in a Norway spruce seed orchard. Forest Genetics 9: 243-255. 
Pakkanen A, T Nikkanen, P Pulkkinen. 2000. Annual variation in pollen contamination and outcrossing in a Picea abies seed orchard. Scandinavian Journal of Forest Research 15: 399-404.

Ritland K, YA El-Kassaby. 1985. The nature of inbreeding in a seed orchard of Douglas-fir as shown by an efficient multilocus model. Theoretical and Applied Genetics 71: 375-384.

Robledo-Arnuncio JJ, L Gil. 2005. Patterns of pollen dispersal in a small population of Pinus sylvestris L. revealed by totalexclusion paternity analysis. Heredity 94: 13-22.

Salaya-Dominguez JM, J López-Upton, JJ Vargas-Hernández. 2012. Variación genética y ambiental en un ensayo de progenies de Pinus patula. Agrociencia 46: 519-534.
SAS Institute (Statistical Analysis System, US). 2002. SAS/ STAT Computer Software. Release 9.00. SAS Institute Inc. Cary.

Sousa VA, HH Hattemer. 2003. Pollen dispersal and gene flow by pollen in Araucaria angustifolia. Australian Journal of Botany 51: 309-317.

Torimaru T, XR Wang, A Fries, B Andersson, D Lindgren. 2009. Evaluation of pollen contamination in an advanced Scots pine seed orchard. Silvae Genetica 58: 262-269.

Wang X, D Lindgren, AE Szmidt, R Yazdani. 1991. Pollen migration into a seed orchard of Pinus sylvestris L. and the methods of its estimation using allozyme markers. Scandinavian Journal of Forest Research 6: 379-385. 
